\title{
Transport Spectroscopy of Single Phosphorus Donors in a Silicon Nanoscale Transistor
}

\author{
Kuan Yen Tan, ${ }^{1,}$ * Kok Wai Chan, ${ }^{1}$ Mikko Möttönen, ${ }^{1,2,3}$ Andrea Morello, ${ }^{1}$ Changyi Yang, ${ }^{4}$ Jessica van Donkelaar, ${ }^{4}$ \\ Andrew Alves, ${ }^{4}$ Juha-Matti Pirkkalainen, ${ }^{1,2}$ David N. Jamieson, ${ }^{4}$ Robert G. Clark, ${ }^{1}$ and Andrew S. Dzurak ${ }^{1}$ \\ ${ }^{1}$ Centre of Excellence for Quantum Computer Technology, School of Electrical Engineering E Telecommunications, \\ University of New South Wales, Sydney NSW 2052, Australia. \\ ${ }^{2}$ Department of Applied Physics/COMP, Helsinki University of Technology, P.O. Box 5100, FI-02015 TKK, Finland. \\ ${ }^{3}$ Low Temperature Laboratory, Helsinki University of Technology, P.O. Box 3500, FI-02015 TKK, Finland. \\ ${ }^{4}$ Centre of Excellence for Quantum Computer Technology, School of \\ Physics, University of Melbourne, Melbourne VIC 3010, Australia.
}

(Dated: October 30, 2018)

\begin{abstract}
We have developed nano-scale double-gated field-effect-transistors for the study of electron states and transport properties of single deliberately-implanted phosphorus donors. The devices provide a high-level of control of key parameters required for potential applications in nanoelectronics. For the donors, we resolve transitions corresponding to two charge states successively occupied by spin down and spin up electrons. The charging energies and the Landé g-factors are consistent with expectations for donors in gated nanostructures.
\end{abstract}

PACS numbers: 73.21.-b,61.72.Vv

The ability to manipulate and measure electrons bound to phosphorus (P) donors is a key ingredient for the realization of quantum information processing schemes using single dopants12! Recent development of metal-oxide-semiconductor(MOS)-compatible nanostructures in silicon ${ }^{3}+6$ together with single-ion detection capabilities $^{7}$ hold promise for the realization of dopantbased spin qubits in silicon ${ }^{8}$. Such qubits are attractive due to the long coherence times of donor electron spins in bulk ${ }^{9}$, but further research is necessary to understand how the donor spin coherence is influenced by the proximity of a $\mathrm{Si}_{-} \mathrm{SiO}_{2}$ interface ${ }^{10111}$. An important step towards this goal is to create gated nanostructures where the energy spectrum of individual donors can be observed and, possibly, manipulated by changing the local environment or the position of the donors with respect to the $\mathrm{Si}-$ $\mathrm{SiO}_{2}$ interface. Pioneering studies of dopant spectroscopy have been carried out on a variety of nanostructures ${ }^{12} 16$, but the presence and location of the donors were uncontrolled. Here, we describe a double-gated nanoscale fieldeffect-transistor (nanoFET) where a chosen mean number of phosphorus donors are deliberately implanted into the conduction channel, and use this device to study the charge and spin states of these individual $\mathrm{P}$ atoms. This structure allows convenient control of four key device parameters: (i) gate-tunable electron density in the source and drain reservoirs ${ }^{5}$; (ii) number of implanted donors ${ }^{7}$; (iii) depth of implanted donors; and (iv) tunnel coupling between donors and reservoirs. In previous studies only the tunnel coupling was controlled by choosing the FET channel length.

The nanoFET studied in this paper [see Figure 1(c)] consists of two independent aluminum gates: a top gate that is biased positively to induce an electron layer that constitutes the source and drain reservoirs; and a barrier gate that depletes the electron layer in the active region of the donors, creating tunnel barriers on each side of the donor potential well. This structure enables the electri-
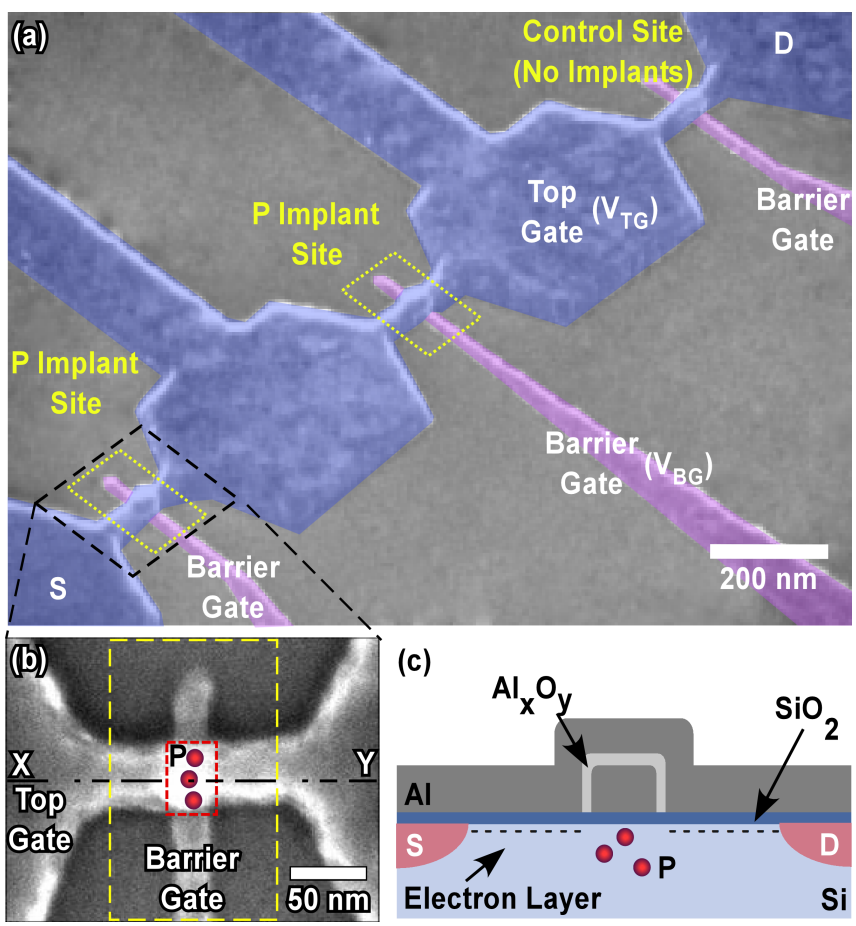

FIG. 1: (Color online) (a) Coloured SEM image of a tribarrier nanoFET. Positive voltage on the top gate induces an electron layer which extends $\sim 25 \mu \mathrm{m}$ to source $(\mathrm{S})$ and drain (D) ohmic contacts. Barrier gates, which are partially underneath the common top gate, allow electrical control of the donor energy levels, for example, the chemical potential can be brought into resonance with Fermi levels of adjacent reservoirs. (b) Zoomed-in SEM image of a single nanoFET device. Yellow dashed line indicates the $\mathrm{P}$ implant window and red dotted line indicates the active region where the mean number of donors is three. (c) (Not to scale) Schematic cross section of a nanoFET along line XY in panel (b). The red source and drain regions are formed by $\mathrm{n}^{+}$diffused contacts. 
cal manipulation of the charge states of the individual phosphorus donors under the barrier gate. In particular, the barrier gate voltage $\left(V_{\mathrm{BG}}\right)$ can be used to tune the donor electrochemical potentials into resonance with those of the source and drain reservoirs, inducing electron transport. The electron density in the source and drain reservoirs can be tuned in-situ by the top gate voltage $\left(V_{\mathrm{TG}}\right)$, while the lithographic width of the barrier gate influences the width, and therefore the transparency, of the tunnel barriers between donor and reservoirs. The excitation spectrum of the donor and its magnetic field dependence extracted from the transport measurements provide important information on electronic properties of donors in close proximity to gate electrodes and induced electron layers.

The devices were fabricated on a high-resistivity $(\sim 10 \mathrm{k} \Omega \mathrm{cm})$ near-intrinsic natural-isotope silicon (100) wafer 17 , with low residual $\mathrm{P}$ background doping $\left(\sim 10^{12}\right.$ $\mathrm{cm}^{-3}$ ). Ohmic contacts for the $\mathrm{n}^{+}$source and drain are phosphorus-diffused regions fabricated using standard UV-lithography and thermal diffusion at temperatures $\sim 950{ }^{\circ} \mathrm{C}$. The $5 \mathrm{~nm}$ gate oxide is high-quality $\mathrm{SiO}_{2}$ grown by dry thermal oxidation. A $5 \mathrm{~s}$ rapid thermal anneal (RTA) at $1000{ }^{\circ} \mathrm{C} \mathrm{N}_{2}$ ambient was carried out to lower the interface trap density to the $2 \times 10^{10} \mathrm{~cm}^{-2} \mathrm{eV}^{-1}$ range, as measured on similarly processed chips $\frac{18}{18}$. For the spatially-selective $\mathrm{P}$ implantation, we used a $150 \mathrm{~nm}$ polymethylmethacrylate (PMMA) layer deposited above the $\mathrm{SiO}_{2}$ as a mask, with $100 \mathrm{~nm} \times 200 \mathrm{~nm}$ apertures defined using electron beam lithography (EBL). In the implantation process, ion acceleration energies of $14 \mathrm{keV}$ and $10 \mathrm{keV}$ were utilized and the flux of the $\mathrm{P}$ ions was controlled to give an average of $N_{d} \sim 3$ individual $\mathrm{P}$ donors in the $50 \mathrm{~nm} \times 30 \mathrm{~nm}$ active area of the device, that is, the region below the overlap of the top and barrier gates [red box in Figure 1(b)]. Subsequently, another RTA at $1000{ }^{\circ} \mathrm{C}$ for $5 \mathrm{~s}$ was applied to activate the donors and repair any damage caused by the implantation process.

The barrier gates used to control the energy levels of the $\mathrm{P}$ donors and to locally deplete the electron layer were fabricated using EBL, thermal evaporation of aluminum, and liftoff. An insulating $\sim 5 \mathrm{~nm} \mathrm{Al}_{x} \mathrm{O}_{y}$ layer was then created using plasma oxidation, where the barrier gates were exposed to a low-pressure oxygen plasma ( 150 mbar) for $3 \mathrm{~min}$ at $150{ }^{\circ} \mathrm{C}$. This $\mathrm{Al}_{x} \mathrm{O}_{y}$ provides electrical insulation between the barrier gates and top gate for voltage differences greater than $\sim 4 \mathrm{~V} 5 \mid 19120$. A second layer of aluminum forming the top gate was again defined using EBL, thermal evaporation, and liftoff. Figure 1 shows a device similar to the ones studied here and its schematic cross-section. Note that there are three independently contacted barrier gates in each device corresponding to three independent devices, for which individual measurements can be done, thus increasing the device yield. The top gate is common to all the barrier regions and the widening of the top gate between barriers ensures that there are no accidental quantum dots created.
A useful feature of our structures is that, when preparing the apertures for ion implantation, we can choose to mask the areas around some of the barrier gates, thus obtaining control devices where we know with certainty that no $\mathrm{P}$ donors are present [see Figure 11(a)].

We measured 27 P-implanted and 11 control devices altogether and present here results from three different devices - one non-implanted control device (Sample A), and two implanted (Samples B and C) - that represent well the qualitative features of the whole batch. Samples $\mathrm{B}$ and $\mathrm{C}$ differ in the energy used for the $\mathrm{P}$ ion implantation (14 keV in $\mathrm{B}, 10 \mathrm{keV}$ in $\mathrm{C}$ ) and in the width of the barrier gate $(30 \mathrm{~nm}$ in $\mathrm{B}, 50 \mathrm{~nm}$ in $\mathrm{C})$. All the experiments presented here were performed in dilution refrigerators at base temperatures $T \lesssim 100 \mathrm{mK}$.

Figure 2(b) shows the source-drain differential conductance of Sample B as a function of the dc source-drain voltage and the barrier gate voltage, with the top gate voltage fixed at $V_{\mathrm{TG}}=3.5 \mathrm{~V}$. We observe the channel turn-on as a function of $V_{\mathrm{BG}}$ when the tunnel barrier between source and drain becomes transparent. In addition, sharp conductance peaks are clearly visible at lower barrier voltages below the threshold. These features are signatures of resonant tunneling through discrete energy levels below the conduction band. Two sets of three different peaks at $0.86 \mathrm{~V}<V_{\mathrm{BG}}<0.93 \mathrm{~V}$ and $0.96 \mathrm{~V}<V_{\mathrm{BG}}<1.03 \mathrm{~V}$ were observed [Figure 2(b)]. These findings are consistent with the expected number of donors $\left(N_{d} \sim 3\right)$ in the active region. However, in other devices we often found only one or two sets of sub-threshold conductance peaks. This is consistent with the Poisson statistics related to the implantation process $^{21}$. In addition, some of the donors can be offcenter in the active region, and couple too strongly to the source (drain) reservoir and too weakly to the drain (source) reservoir. The total conductance is then dominated by the slowest tunnel rate, making certain donors immeasurable in transport even if they are physically present in the channel.

We identify peaks $a_{1}, b_{1}$, and $c_{1}$ as corresponding to the transition from the positively charged state $D^{+}$to the neutral state $D^{0}$ of each donor, whereas peaks $a_{2}, b_{2}$, and $c_{2}$ correspond to a transition to the charged state $D^{-}$. The peaks associated with the same donor have almost the same lever arm factors $\alpha=C_{\mathrm{BG}} / C_{\Sigma}$, where $C_{\Sigma}=C_{\mathrm{BG}}+C_{\mathrm{S}}+C_{\mathrm{D}}+C_{\mathrm{TG}}+C_{0}$ and $C_{\mathrm{BG}}, C_{\mathrm{TG}}, C_{\mathrm{S}}$, and $C_{\mathrm{D}}$ are the coupling capacitances between the corresponding donor and the barrier gate, top gate, source, and drain, respectively. The self-capacitance of the donor is denoted by $C_{0}$. This $\alpha$-factor can be determined from the positive and negative slopes of each conductance peak in the differential conductance plot [Figure 2(b)], where the positive slope is given by $C_{\mathrm{BG}} /\left(C_{\Sigma}-C_{\mathrm{S}}\right)$ and the negative slope equals $C_{\mathrm{BG}} / C_{\mathrm{S}}$ (with drain grounded) 12 .

Figure 2(b) shows that the slopes of peaks $b_{1}$ and $b_{2}$ are similar to each other, with $\alpha=0.45$, and hence they are attributed to the same donor. Multiplying $\alpha$ by the difference in barrier gate voltage $\Delta V_{\mathrm{BG}}=62 \mathrm{mV}$ between $b_{1}$ 

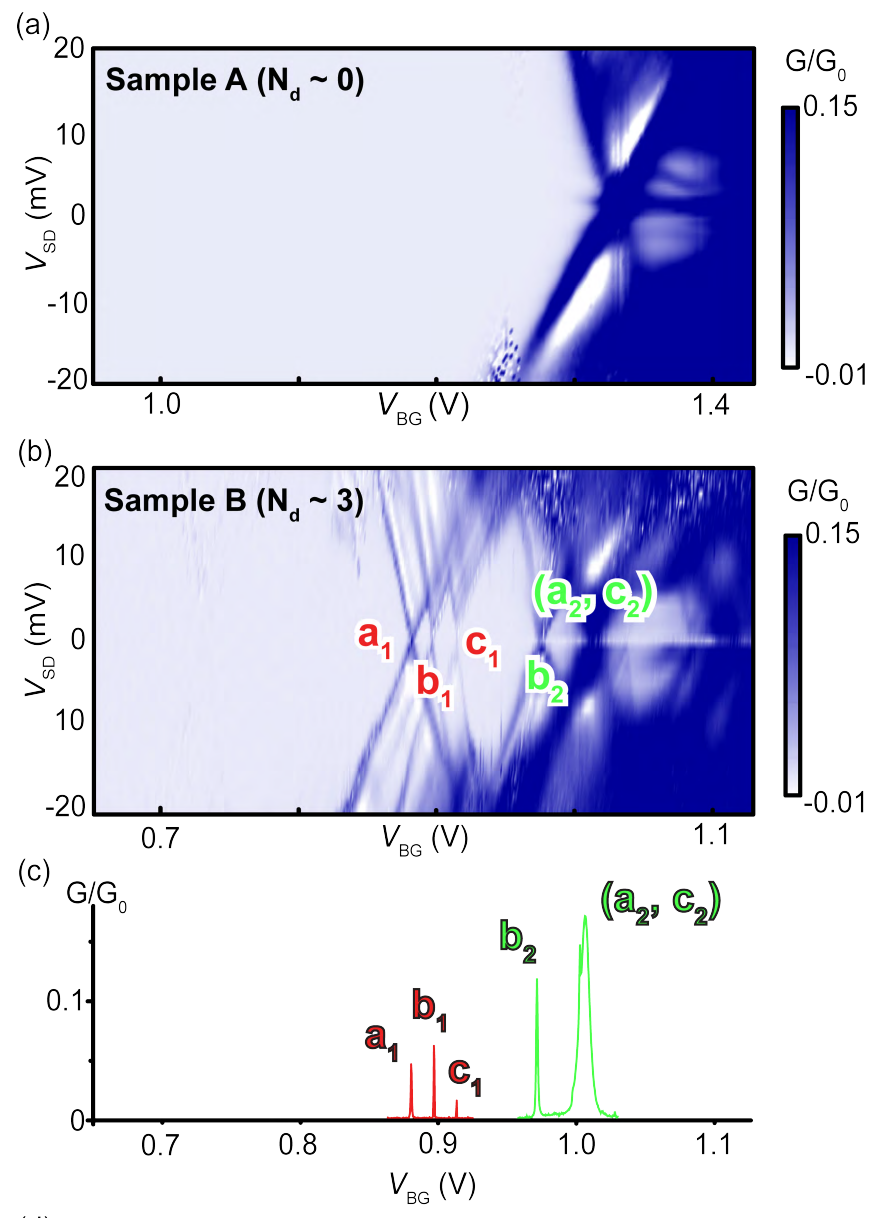

(d)

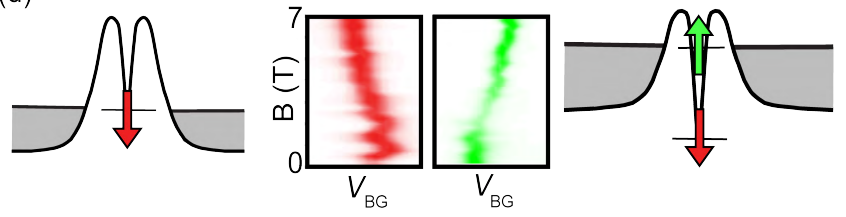

FIG. 2: (Color online) (a) Stability diagram showing differential conductance as a function of barrier gate and dc sourcedrain (SD) bias of a control device (Sample A) with no implanted donors. (b) Stability diagram of Sample B with six labeled peaks. (c) A cut of the stability diagram in panel (b) at $V_{\mathrm{SD}}=0 \mathrm{~V}$, highlighting the resonant tunneling peaks due to three donors. (d) Direction of peak shifts under global magnetic field up to 7 T. Peak $a_{1}$ (red) shifted with the field to the left while peak $b_{2}$ (green) shifted to the right. Schematics indicate that $a_{1}$ is due to tunneling of spin-down electrons and $b_{2}$ is a two-electron singlet state. All data in this figure were measured at $T \lesssim 100 \mathrm{mK}$, and $G_{0}=e^{2} / h$.

and $b_{2}$ yields the $D^{0}$ to $D^{-}$charging energy for donor $b$, $E_{c}=28 \pm 3 \mathrm{meV}^{22}$. Peaks $a_{2}$ and $c_{2}$ are just barely resolvable in Figure 2(c), so we cannot make a specific match with either of the $D^{0}$ peaks $a_{1}$ and $c_{1}$. However, we can use the slope $\alpha=0.26$ of peak $a_{1}$, together with the gate voltage difference $\Delta V_{\mathrm{BG}}=131 \mathrm{mV}$ between peaks $a_{1}$ and $\left(a_{2}, c_{2}\right)$, to calculate the charging energy for donor $a$ to be $E_{c}=34 \pm 6 \mathrm{meV}$. In both cases $E_{c}$ is lower than the $44 \mathrm{meV}$ expected for phosphorus in bulk ${ }^{23 \mid 24}$ due to the fact that there is a substantial capacitive coupling of the donor to the surrounding electrodes 12113 . Across the entire series of implanted devices we have observed $E_{c}$ ranging from $21 \mathrm{meV}$ to $39 \mathrm{meV}$, with an average value of $28 \mathrm{meV}$. A simple model of the gate electrodes and the donor at the mean implantation depth in silicon 25126 provides a rough estimate of the capacitances required to calculate the charging energy $E_{c}=e^{2} / C_{\Sigma}$. With a donor depth $d=5-20 \mathrm{~nm}$ below the $\mathrm{Si}-\mathrm{SiO}_{2}$ interface, the model yields $E_{c}=39-40 \mathrm{meV}$. The discrepancy with the measured values can be, for example, due to the limitations of a simple capacitance model for this ultra-small few-electron system.

Magnetospectroscopic studies of Sample B are shown in Figure 2(d), obtained by applying a magnetic field up to $7 \mathrm{~T}$ in the plane of the electron layer. As the magnetic field was increased, peaks $a_{1}$ and $c_{1}$ shifted to lower values of $V_{\mathrm{BG}}$ whereas the peak $b_{2}$ shifted to higher values ${ }^{27}$. The direction of these shifts provides information about the spin-polarity, relative to the external field, of the electrons that contribute to the tunneling current: a shift to lower (higher) $V_{\mathrm{BG}}$ corresponds to tunneling of spin-down (-up) electrons. The magnetic-field-dependent shifts are consistent with the assignment of peaks $a_{1}$ and $c_{1}$ to $D^{0}$ and $b_{2}$ to $D^{-}$states, as inferred from the bias spectroscopy in Figure 2(b). This is due to the fact that electrons in $D^{-}$, being a two-electron ground state, form a singlet with opposing electron spins ${ }^{28}$. A quantitative analysis of the magnetic field dependence is given for Sample C below.

As a control, another nanoFET structure (Sample A) was fabricated on the same substrate but with no implanted dopants in the active region. The stability diagram is shown in Figure 2(a), with $V_{\mathrm{TG}}=2.0 \mathrm{~V}$. It is significantly different from the data obtained for devices with donors in the active region. In particular, there are no sharp resonant tunneling features with large charging energies well below threshold. A weak minimum is observed in the threshold region $\left(1.32 \mathrm{~V}<V_{\mathrm{BG}}<1.40 \mathrm{~V}\right)$ which is most likely due to the formation of an unintentional dot, due to weak local disorder in the barrier region. This is consistent with the small charging energy $(<10 \mathrm{meV})$ and the large width of the peaks, which implies transparent tunnel barriers. Features reminiscent of Kondo-enhanced conductance $e^{29} \sqrt{31}$ in this region, plus the absence of other peaks at lower $V_{\mathrm{BG}}$, suggest that this could be the first electron in such a dot. The control data reinforce the interpretation that the peaks observed in Sample B are due to resonant tunneling through the deliberately implanted $\mathrm{P}$ donors. We note that some control devices processed without a final forming gas anneal (FGA) showed sub-threshold conductance features which could be attributed to localized states associated with interface traps, for example. These features were clearly distinguishable from donors due to their much lower charging energies, typically only up to $13 \mathrm{meV}$. We found that a FGA can reduce significantly the charge 
trap density as well as the charge noise ${ }^{5}$.

(a)

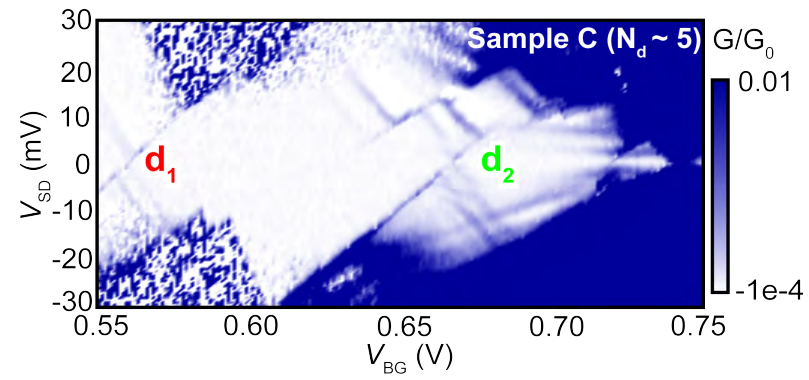

(b)

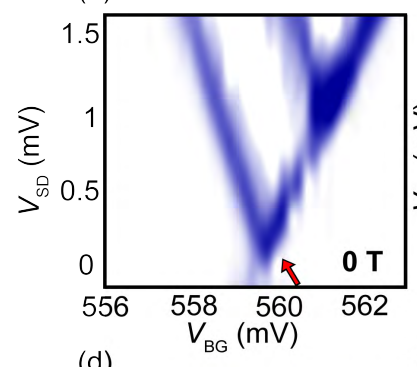

(c)

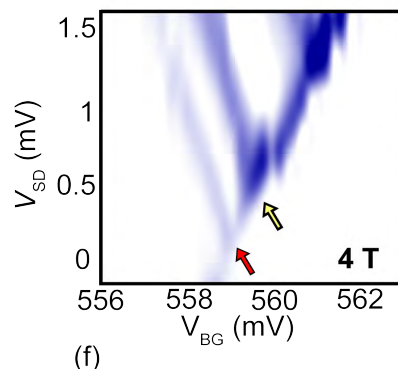

(f)

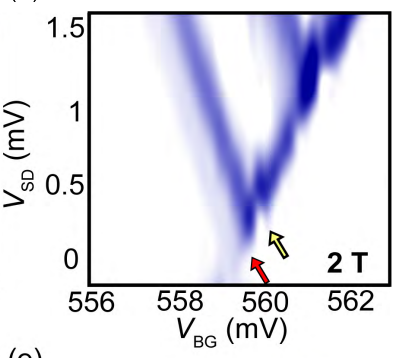

(e)

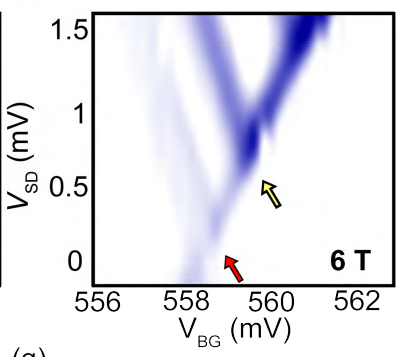

(g)
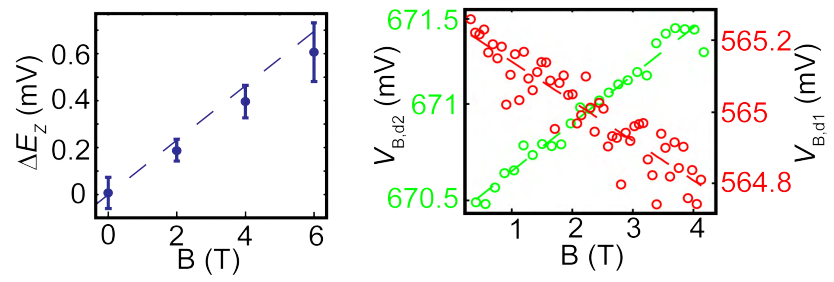

FIG. 3: (Color online) (a) Bias spectroscopy showing differential conductance as a function of barrier gate and dc SD-bias of Sample C. (b)-(e) Detailed bias spectroscopies of peak $d_{1}$ at $B=0,2,4$, and $6 \mathrm{~T}$. Red (yellow) arrows indicate spin-down (-up) states entering the bias window. (f) Energy splitting $\left(\Delta E_{Z}\right)$ of the spin-up and spin-down states deduced from panels (b) $-(\mathrm{e})$. The dashed line indicates $2 \mu_{B} B / e$ for reference, where $\mu_{B}$ is the Bohr magneton. (g) Positions of the peaks $d_{1}$ (red circles) and $d_{2}$ (green circles) in the barrier gate voltage as a function of global magnetic field. The dashed lines are linear fits to the data points.

Sample C was fabricated using the same processes as Sample B, with the exception that the phosphorus donors were implanted with $10 \mathrm{keV}$ ion-beam acceleration energy, and the barrier gate was $50 \mathrm{~nm}$ wide, yielding weaker tunnel coupling between each donor and the reservoirs, and accordingly sharper conductance features. With $10 \mathrm{keV}$ implantation energy, the donors are ex- pected to be $\sim 12 \mathrm{~nm}$ from the $\mathrm{Si}-\mathrm{SiO}_{2}$ interface ${ }^{26}$, thus closer to the gate compared with the donors implanted with $14 \mathrm{keV}$ beam energy in Sample B. The bias spectroscopy of Sample C is presented in Figure 3(a). Here, only one pair of $D^{0}$ and $D^{-}$states, denoted by $d_{1}$ and $d_{2}$, is strongly visible although the mean number of donors under the active region (here $50 \times 50 \mathrm{~nm}^{2}$ ) is $N_{d} \sim 5$. This is not surprising because the wider tunnel barriers, as compared with Sample B, make the requirement of the donors being well-centered even more stringent ${ }^{32}$.

In the Coulomb blockaded region between $d_{1}$ and $d_{2}$, additional faint conductance features were observed at finite source-drain bias. These features are attributed to nearby weakly coupled donors or other charge traps 33 . We also observed random telegraph signals (RTS) in the conductance in well-defined regions at $\left|V_{\mathrm{SD}}\right|>10 \mathrm{mV}$. This phenomenon most likely arises because the electrochemical potential (and thus the conductance) of the visible donor depends on the charge state of the surrounding donors or traps, due to mutual charging energy effects. Random switching of weakly coupled charge centers can then give rise to the observed RTS. Thus the donor can be possibly used as a charge sensor for the nearby charge centers 34 . Our preliminary theoretical analysis shows that the distance between the visible donor and the nearby donor or charge trap is more than $25 \mathrm{~nm}^{35}$.

The sharpness of the conductance peaks in the widebarrier Sample C allowed us to study in more detail the spin states of the donor. Figures $3(\mathrm{~b}-\mathrm{e})$ show a set of bias spectroscopy data on the peak $d_{1}$ of Sample C, with applied magnetic fields $B=0,2,4,6 \mathrm{~T}$. For $B>2 \mathrm{~T}$ we clearly resolve an excited state at finite bias, which we attribute to the excited Zeeman state (spin-up) of the first electron on the donor. The peaks in the differential conductance due to spin-down and -up electrons are indicated by red and yellow arrows, respectively. The separation of these peaks on the $V_{\mathrm{BG}}$ axis multiplied by $\alpha$ should then equal the Zeeman splitting of the $D^{0}$ spin states. To verify this, we fitted Gaussian functions to the differential conductance peaks in Figures 3(b-e) at various fixed SD bias points to obtain an accurate measure of their position. The resulting energy splitting $\Delta E_{Z}$ is plotted as a function of magnetic field in Figure 3(f), together with error bars obtained from the fitting procedure and the error of $\alpha$ (including $\Delta E_{Z}$ at $B=0$ ). As shown in Figure 3 (f), $\Delta E_{Z}$ is in good agreement with the Zeeman splitting for a Landé $g$-factor $g=2$, as expected for an electron bound to a hydrogenic donor potential.

Figure $3(\mathrm{~g})$ shows the Zeeman shifts of peaks $d_{1}$ and $d_{2}$ in barrier gate voltage. As expected, the peaks shifted linearly in opposite directions, but the magnitudes of the shifts are different. The peak $d_{1}$ shifted an average of $-24 \mu \mathrm{VT}^{-1}$ whereas the peak $d_{2}$ shifted an average of 95 $\mu \mathrm{VT}^{-1}$, yielding individual $g$-factors far from 2 . However, if the difference of the shifts for $d_{1}$ and $d_{2}$ is considered, we obtain the correct value $g=2.4 \pm 0.5$. The asymmetry in the peak shifts can be caused, for example, by a slow global drift in the electric potentials in the 
device over time.

While the nanoFET devices in this study were used to investigate phosphorus donors, we note that these double-gated structures could be used to study discrete energy states of any donor or acceptor atom in silicon. In the latter case, this could be achieved using a pMOS nanoFET, employing $\mathrm{p}^{+}$source-drain contacts and a negative gate voltage. By employing on-chip waveguides or microwave cavities to create a magnetic field in resonance with the spin split $D^{0}$ donor state, one could use these structures for the study of electrically-detected magnetic resonance of single donor spins, as recently demonstrated for small ensembles ${ }^{36}$.

In conclusion, we have shown that resonant tunneling of electrons through discrete energy states of phosphorus donors in silicon can be observed in planar nanoscale FET structures, where the presence and depth of the donors is deliberately chosen, and basic device parameters such as tunnel couplings and electron density can be conveniently controlled. We have identified the $D^{0}$ and $D^{-}$charge states of individual donors, measured their charging energy, and observed the Zeeman shift of their spin states in magnetic fields up to $7 \mathrm{~T}$. Control devices, deliberately fabricated with no donors in the channel, showed markedly different transport behavior than the characteristic features present in donor-implanted devices. These experiments provide important understanding of the electric and magnetic properties of individual phosphorus donors needed for the realization of Si:P nanoelectronics. Moreover, these structures are compatible with single-ion implant detectors ${ }^{737}$ thus enabling devices with precisely one implanted donor. The demonstrated ability to resolve spin states of donors in these devices could also enable the measurement of electricallydetected magnetic resonance for a single-donor electron spin.

The authors thank D. Barber and R. P. Starrett for technical support in the National Magnet Laboratory, UNSW, E. Gauja for assistance in the UNSW Semiconductor Nanofabrication Facility, and A. Cimmino and R. Szymanski for technical support at the University of Melbourne. We also acknowledge J. McCallum, L. C. L. Hollenberg, C. C. Escott and M. Eriksson for useful discussions. M. M. and J.-M. P. acknowledge Academy of Finland and Emil Aaltonen Foundation for financial support. This work is supported by the Australian Research Council Centre of Excellence scheme, the Australian Government, the U.S. National Security Agency (NSA), and the U.S. Army Research Office (ARO) (under Contract No. W911NF-08-1-0527).
* Electronic address: kuan-yen@student.unsw.edu.au

1 B. Kane, Nature 393, 133 (1998).

2 L. C. L. Hollenberg, A. D. Greentree, A. G. Fowler, and C. J. Wellard, Phys. Rev. B. 74, 045311 (2006).

3 S. D. Lee, S. J. Shin, S. J. Choi, J. J. Lee, J. B. Choi, S. Park, S.-R. E. Yang, S. J. Lee, and T. H. Zyung, Appl. Phys. Lett. 89, 023111 (2006).

4 A. Fujiwara, H. Inokawa, K. Yamazaki, H. Namatsu, Y. Takahashi, N. M. Zimmerman, and S. B. Martin, Appl. Phys. Lett. 88, 053121 (2006).

5 S. J. Angus, A. J. Ferguson, A. S. Dzurak, and R. G. Clark, Nano Letters 7, 2051 (2007).

${ }^{6}$ H. Liu, T. Fujisawa, H. Inokawa, Y. Ono, A. Fujiwara, and Y. Hirayama, Appl. Phys. Lett. 92, 222104 (2008).

7 D. N. Jamieson, C. Yang, T. Hopf, S. M. Hearne, C. I. Pakes, S. Prawer, M. Mitic, E. Gauja, S. E. Andresen, F. E. Hudson, et al., Appl. Phys. Lett. 86, 202101 (2005).

8 A. Morello, C. C. Escott, H. Huebl, L. H. Willems van Beveren, L. C. L. Hollenberg, D. N. Jamieson, A. S. Dzurak, and R. G. Clark, arXiv:0904.1271 (unpublished).

9 A. M. Tyryshkin, S. A. Lyon, A. V. Astashkin, and A. M. Raitsimring, Phys. Rev. B 68, 193207 (2003).

10 T. Schenkel, J. A. Liddle, A. Persaud, A. M. Tyryshkin, S. A. Lyon, R. de Sousa, K. B. Whaley, J. Bokor, J. Shangkuan, and I. Chakarov, Appl. Phys. Lett. 88, 112101 (2006).

11 R. de Sousa, Phys. Rev. B. 76, 245306 (2007).

12 H. Sellier, G. P. Lansbergen, J. Caro, S. Rogge, N. Collaert, I. Ferain, M. Jurczak, and S. Biesemans, Phys. Rev. Lett. 97, 206805 (2006).

13 G. P. Lansbergen, R. Rahman, C. J. Wellard, I. Woo, J. Caro, N. Collaert, S. Biesemans, G. Klimeck, L. C. L.
Hollenberg, and S. Rogge, Nature Phys. 4, 656 (2008).

14 L. E. Calvet, J. P. Snyder, and W. Wernsdorfer, Phys. Rev. B (2008).

15 L. E. Calvet, R. G. Wheeler, and M. A. Reed, Phys. Rev. Lett. 98, 096805 (2007).

16 L. E. Calvet, R. G. Wheeler, and M. A. Reed, Phys. Rev. B 76, 035319 (2007).

17 Topsil high-purity silicon (HPS) wafers were used http://www.topsil.com.

18 J. McCallum, M. Dunn, and E. Gauja, Mater. Res. Soc. Symp. Proc. 1074 (2008).

19 P. C. Heij, Ph.D. Thesis, Delft University of Technology pp. $14-16$ (2001).

20 W. H. Lim, H. Huebl, L. H. W. van Beveren, S. Rubanov, P. G. Spizzirri, S. J. Angus, R. G. Clark, and A. S. Dzurak, Appl. Phys. Lett. 94, 173502 (2009).

21 Explicitly, for $n=3$, we find $\mathrm{P}(0)=0.04, \mathrm{P}(1)=0.14$, $\mathrm{P}(2)=0.23, \mathrm{P}(3)=0.24, \mathrm{P}(4)=0.16, \mathrm{P}(5)=0.10$.

22 This treatment neglects the mutual charging effects between the donor and other localized charges but should yield a good estimate here.

23 A. K. Ramdas and S. Rodriguez, Reports on Progress in Physics 44, 1297 (1981).

24 M. Taniguchi and S. Narita, Solid State Communications 20, 131 (1976).

25 The software used to extract coupling capacitances is FAstcAP, K. Nabors and J. White, IEEE Trans. Comput.Aided Des. 10, 1447. (1991).

26 Stopping and range of ions in matter (SRIM) software was used to determine nominal phosphorous donor depth into silicon.

27 Due to the large width of the closely adjacent peaks $\left(a_{2}\right.$ and 
$c_{2}$ ) it was not possible to measure any shift with magnetic field, while peak $b_{1}$ showed an irregular non-monotonic shift.

28 H.-A. Engel and D. Loss, Phys. Rev. Lett. 86, 4648 (2001).

${ }^{29}$ D. Goldhaber-Gordon, H. Shtrikman, D. Mahalu, D. Abusch-Magder, U. Meirav, and M. A. Kastner, Nature 391, 156 (1998).

30 J. R. Hauptmann, J. Paaske, and P. E. Lindelof, Nat. Phys 4, 373 (2008).

31 An extensive study of Kondo effects in silicon nanoMOSFETs is in preparation by G.P. Lansbergen, G.C. Tettamanzi, J. Verduijn, N. Collaert, S. Biesemans, M. Blaauboer and S. Rogge.

32 When the resistances of the tunnel barriers are very high, any further widening of one of the barriers - due to the donor being off-center - would make the tunnel current immeasurably small.

33 M. Pierre, M. Hofheinz, X. Jehl, M. Sanquer, G. Molas, M. Vinet, and S. Deleonibus, arXiv:0810.0672 (2008).

34 M. Xiao, I. Martin, E. Yablonovitch, and H. Jiang, Nature 430, 435 (2004).

35 A detailed study of the mutual charging effects will be published elsewhere.

36 C. C. Lo, J. Bokor, T. Schenkel, A. M. Tyryshkin, and S. A. Lyon, Appl. Phys. Lett. 91, 242106 (2007).

37 C. D. Weis, A. Schuh, A. Batra, A. Persaud, I. W. Rangelow, J. Bokor, C. C. Lo, S. Cabrini, E. SiderasHaddad, G. D. Fuchs, et al., J. Vac. Sci. Technol. B 26, 2596 (2008). 